\title{
Spontaneous bacterial peritonitis due to Campylobacter jejuni
}

\author{
N. I. MCNEIL \\ M.D., M.R.C.P. \\ S. BUTtoo \\ M.B., B.S. \\ G. L. RIDGWAY
M.D., M.R.C.Path. \\ Departments of Medicine and Microbiology, University College Hospital, London WC1
}

\begin{abstract}
Summary
A patient with alcoholic cirrhosis complicated by ascites developed spontaneous bacterial peritonitis. Campylobacter jejuni-(a microaerophilic bacillus) - was isolated from ascitic fluid and blood.
\end{abstract}

KEY WORDS: liver cirrhosis, alcoholic, bacteraemia.

\section{Introduction}

Spontaneous bacterial peritonitis is a well-established complication of hepatic cirrhosis (Conn and Fessel, 1971). Though a wide range of causative organisms have been described, these are almost entirely aerobic. We report a case of spontaneous bacterial peritonitis due to Campylobacter jejuni, a microaerophilic bacillus.

\section{Case report}

A 56-year-old man, with a long history of alcoholrelated disease including alcoholic hepatitis on three occasions, presented with lethargy, vomiting and weakness of 2 weeks' duration. He had been vomiting 2-3 times daily and in the days before admission altered blood was present. Ankle and abdominal swelling had been present for about 8 weeks. He was jaundiced with massive ascites and peripheral oedema, as well as other features of chronic liver disease.

Having vomited altered blood in hospital, endoscopy showed gastrointestinal bleeding to come from oesophagitis which was subsequently treated with antacids and ranitidine. Ascitic fluid examination immediately after admission was normal with no growth. He was oliguric and rapidly became encephalopathic, treated with intravenous fluids and plasma, and with neomycin and lactulose respectively.
Three days after admission the development of worsening confusion and increasing girth was an indication for repeat ascitic fluid aspiration. He was apyrexial and had only mild abdominal pain. Clear yellow fluid with a cell count of $12 \times 10^{9} / 1(92 \%$ neutrophils) was obtained. Amoxycillin, $2 \mathrm{~g}$ daily in divided doses, was started immediately. Both ascitic fluid and blood cultures, taken simultaneously, grew Campylobacter jejuni. Faecal culture was not performed.

The organism was identified as $C$. jejuni on the grounds of morphology, positive oxidase reaction, positive hippurate test and in vitro sensitivity to nalidixic acid. The identification was confirmed by a reference laboratory.

Gentamicin, to which the organism was sensitive, was started, $120 \mathrm{mg}$ initially, then as indicated by blood levels. However renal function continued to deteriorate from admission to hospital, and was not improved by careful management of his circulating volume. He became more confused. Jaundice deepened with no evidence of biliary obstruction. After 16 days he suffered continuous epistaxis requiring a nasal pack. The next day he passed melaena, became extremely hypotensive and died.

Post-mortem examination demonstrated micronodular cirrhosis. Multiple gastric erosions were present. The gall bladder contained pigment stones but the biliary tract was patent.

\section{Discussion}

This is the first report to our knowledge of spontaneous bacterial peritonitis being caused by $C$. jejuni. The clinical presentation of increasing confusion without fever, and the findings on examination of the ascitic fluid were typical of spontaneous bacterial peritonitis, which has a mortality of $70-80 \%$ in patients with alcoholic cirrhosis (Conn 
and Fessel, 1971; Weinstein et al., 1978). The infecting organisms are frequently isolated from blood as well as ascitic fluid, as in this case. However, aerobes are almost always isolated (Targan, Chow and Guze, 1977), and the organisms are generally derived from the intestinal flora. The paucity of anaerobes is thought to be related to firstly the oxygen tension of ascitic fluid, in cirrhotics approximately that of mixed venous blood, and secondly the requirement for the infecting organism to pass through the oxygenated intestinal wall (Sheckman, Onderdonk and Bartlett, 1977).

The seriousness of bacterial peritonitis requires that a search be made for anaerobic, microaerophilic and aerobic bacteria so that appropriate therapy can be initiated as soon as possible.

\section{Acknowledgment}

We are grateful to Dr M. B. Skirrow, Worcester Royal Infirmary, for confirmation of the organism identification.

\section{References}

CONN, H.O. \& FESSEL, J.M. (1971) Spontaneous bacterial peritonitis in cirrhosis: variations on a theme. Medicine, 50, 161.

ShECKMAN, P., ONDERDONK, A.B. \& BARTLETT, J.G. (1977) Anaerobes in spontaneous peritonitis. Lancet, ii, 1223.

TARGAN, S.R., CHOW, A.W. \& GUZE, L.B. (1977) Role of anaerobic bacteria in spontaneous peritonitis of cirrhosis. American Journa 5 of Medicine, 62, 397.

Weinstein, M.P., IANnini, P.B., Stratton, C.W. \& Eickhoff, T.C. (1978) Spontaneous bacterial peritonitis. American Journal of Medicine, 64, 592.

(Accepted 1 June 1983) 